\title{
Molecular imaging of tumor-specific markers and their expression in other organs
}

\author{
Diwakar Jain, MD, FACC, MASNC ${ }^{\mathrm{a}}$ \\ a Cardio-Oncology Service, Nuclear Cardiovascular Imaging Laboratory, Department of Cardi- \\ ology, Westchester Medical Center, New York Medical College, Valhalla, NY
}

Received Jun 2, 2020; accepted Jun 2, 2020

doi: $10.1007 / \mathrm{s} 12350-020-02310-1$

\section{See related article, pp. 812-821}

Nuclear imaging provides us with a unique ability to study various biochemical, metabolic and molecular processes, expression of cell membrane proteins and receptors, matrix composition, and gene expression, at a cellular, tissue and organ level in intact animals and humans under various physiological conditions. ${ }^{1-6}$ This involves an identification of a target inside the body, which is highly specific for a particular disease process and is abundantly expressed and is amenable to an exogenously administered probe. The next step requires an identification or development of new ligand(s) specific for that particular target and its radiolabeling with a suitable radiotracer to image the target. These techniques allow us to study the metabolism of glucose, free fatty acids and various amino acids; nucleic acid synthesis as a marker of cell proliferation; activity of membrane transporters; expression of cell membrane associated and matrix-associated proteins and receptors and delivery of therapeutic interventions to these targets. These imaging techniques have greatly enhanced our understanding of tumor biology and pathophysiology and have also played a significant role in developing several highly effective novel cancer therapies. ${ }^{7}$ Fluorine-18 labeled deoxyglucose $\left({ }^{18} \mathrm{FDG}\right)$ imaging has been the backbone of molecular imaging and is in extensive clinical use for diagnosing and staging of solid cancers

Reprint requests: Diwakar Jain, MD, FACC, MASNC, Cardio-Oncology Service, Nuclear Cardiovascular Imaging Laboratory, Department of Cardiology, Westchester Medical Center, New York Medical College, 100 Woods Road, Valhalla, NY 10595. dj2700@gmail.com

J Nucl Cardiol 2021;28:822-4.

$1071-3581 / \$ 34.00$

Copyright (C) 2020 American Society of Nuclear Cardiology. and in evaluating response to therapy in patients with various malignancies. ${ }^{8}{ }^{18}$ FDG imaging has also provided an interesting insight into the tumor microenvironment: a highly hypoxic and acidic milieu, primarily dependent upon anerobic glycolysis for meeting its energy demand. This requires a profound increase in glucose uptake, facilitated by an upregulation of cell membrane GLUT transporters and an upgraded system for clearance of lactic acid and other waste metabolites in the cancer cells. Although, ${ }^{18}$ FDG remains the key radiotracer used in oncology, a number of other radiotracers such as ${ }^{18} \mathrm{~F}$-FLT (fluorothymidine), ${ }^{18}$ F-Methionine, ${ }^{18}$ F-fluoroestrodiol have been developed and are undergoing evaluation for studying various aspects of tumor biology. ${ }^{9-11}$

In this issue of the journal Siebermair et al present cardiac findings in 32 patients with cancers (pancreatic cancer in 18 and malignancies of other organ systems in 14), who underwent whole body PET-CT or PET-MR imaging with ${ }^{68} \mathrm{Ga}-\mathrm{FAPI}$ (fibroblast activation protein inhibitor), a quinoline based ligand with high avidity for fibroblast activation protein(FAP) radiolabeled with Gallium-68. This imaging was a part of theranostic imaging study to identify patients with tumors with high expression of FAP, who can be targeted with therapeutic doses of FAPI. ${ }^{12}$ The authors report interesting cardiac findings in this otherwise oncological imaging study.

A brief background of the fibroblasts and their relationship to cancers is warranted before discussing the relevance of this study. Fibroblasts are the most common cell type in the extracellular matrix in the body, and are responsible for collagen and other matrix protein synthesis, tissue homeostasis, and facilitate cell communication and function in the normal tissues. Solid cancers also require fibroblasts to provide the matrix for their growth and invasiveness. ${ }^{13}$ Several factors secreted from cancer cells or immune cells, such as transforming growth factor beta (TGF- $\beta$ ), platelet derived growth factor (PDGF), hepatocyte growth factor (HGF) and 
fibroblast growth factor (FGF), metalloproteinases and reactive oxygen species can activate normal fibroblasts and recruit them for cancer cells. These activated and recruited fibroblasts are known as cancer-associated fibroblasts (CAF). Whereas cancer cells initiate and sustain CAF, CAF become an integral part of cancer growth, motility and invasion. They facilitate tumor progression, metastasis and chemoresistance. Cancer cells and CAF establish a partnership, which is further fueled by inflammatory cells. An understanding of this relationship provides novel opportunities and targets for cancer therapy. Upon initial activation by cancer cells, fibroblasts undergo a phenotype switch to become CAF and express various markers which are not present in the normal fibroblasts. Fibroblast activation protein (FAP) a membrane-bound glycoprotein is one of these markers. FAP is a transmembrane glycoprotein with serine protease activity. This protein is closely related to dipeptidyl peptidases (DPP-4, DPP8 and DPP9). FAP is most closely related to DPP-4 (approximately 50\% of their amino acids are identical). The active site of FAP is localized in the extracellular part of the protein and has a dipeptidase and an endopeptidase activity. Several bioactive peptides and structural proteins are physiological FAP substrates. ${ }^{13-15}$ FAP is expressed during embryonic development, but its expression under physiological conditions is very low in the adult tissues. However, FAP expression is high in the CAF of solid cancers and in granulation tissue of healing wounds. FAP plays a role in the control of fibroblast growth and progression of cancers. FAP over-expression is seen in over $90 \%$ of all human carcinomas and is an important therapeutic target. Several therapeutic agents are currently targeting FAP in various cancers including antiFAP antibodies, FAP antibody-drug conjugates, FAPCAR T cells, FAP vaccines, and small molecules with avidity for FAP. Understanding the mechanisms employed by the cancer cells for immune evasion and surveillance and its targeting by monoclonal antibodies has resulted in the development of highly powerful and effective therapies for the treatment of cancers. ${ }^{16}$ Similarly, a better understanding of the mechanism(s) employed by the tumors to induce a phenotypic change in normal fibroblasts and vascular endothelial cells to partner with tumors, may provide very effective targets for interruption of tumor growth. Imaging agents like ${ }^{68} \mathrm{Ga}-\mathrm{FAPI}$ can play an important role in this direction.

Now returning back to the study by Siebermair et al, abnormal cardiac uptake was observed co-incidentally in 6 of their 32 patients (localized myocardial uptake in 3 and diffuse myocardial uptake in the remaining 3), whereas no discernible uptake was observed in the remaining 26. On comparing these groups, patients with increased cardiac ${ }^{68} \mathrm{Ga}$-FAPI uptake were older, and had lower LVEF. Furthermore, localized myocardial FAPI uptake corresponded to the site of prior MI or percutaneous interventions in 3 of these 6 patients. None of the patients with no cardiac uptake had prior myocardial infarction. Whereas a limited sample size, precludes any definite conclusions, yet a few preliminary interesting observations can be made. Firstly, this study underscores the importance of paying attention to all organ systems particularly cardiovascular system while evaluating newer molecular imaging modalities in patients with cancers. Various molecular pathways can be activated in the tumors and heart or other organ systems either through different routes or by a shared pathophysiology. ${ }^{18}$ FDG imaging detects tumors and ischemic/hibernating myocardium and myocardial inflammation by imaging enhanced glucose uptake induced by very different pathways. Secondly, some of the cancer imaging probes may also find important applications in understanding cardiovascular pathophysiology. An ability to image the process of fibroblast activation in the myocardium following ischemia, radiation, inflammation and exposure to cardiotoxic cancer therapy may enhance our understanding of the complex interaction between myocardial healing, fibrosis, scarring, remodeling and left ventricular dysfunction. This may also provide newer targets for therapeutic interventions for myocardial regeneration and recovery of cardiac function in patients with systolic and diastolic dysfunction. Perhaps, the findings of this study would promote an interest in paying closer attention to the heart and other organ systems and a greater multi-disciplinary collaboration across imaging experts in future molecular imaging.

\section{Disclosure}

Diwakar Jain declares that he has no conflicts of interest to disclose.

\section{References}

1. Jain D, Ghanbarinia A, He ZX. Developing a new PET myocardial perfusion tracer (editorial). J Nucl Cardiol 2009;16:689-90. http s://doi.org/10.1007/s12350-009-9113-X.

2. Jain D, He ZX, Lele V. Cardiac hot spot imaging with (18)FDG. Semin Nucl Med 2014;44:375-85. https://doi.org/10.1053/j.semn uclmed.2014.06.010.

3. Jain D. Cardiac adrenergic neuronal activity, sleep apnea and potential therapeutic role of nocturnal ventilatory assistance in patients with heart failure (editorial). J Nucl Cardiol 2018. http s://doi.org/10.1007/s12350-018-1234-7.

4. Jain D, He ZX. Direct myocardial ischemia imaging with exercise (18)FDG. J Nucl Cardiol 2015;22:111-4. https://doi.org/10.1007/ s12350-014-0001-7. 
5. Harikrishnan $\mathrm{P}$, Gerard $\mathrm{P}$, Jain D. ${ }^{18} \mathrm{~F}-\mathrm{FDG}$ for imaging microvascular injury (editorial). J Nucl Cardiol 2018;25:441-2. h ttps://doi.org/10.1007/s12350-017-0955-3.

6. Joseph B, Bhargava KK, Kandimala J, Malhi H, Afriyie M, Volenberg I, et al. The nuclear imaging agent sestamibi is a substrate for both MDR1 and MDR2 p-glycoprotein genes. Eur J Nucl Med 2003;30:1024-31.

7. Verhoeven M, Seimbille Y, Dalm SU. Therapeutic applications of pretargeting. Pharmaceutics 2019;11:434. https://doi.org/10.3390/ pharmaceutics11090434.

8. Fonti R, Conson M, Del Vecchio S. PET/CT in radiation oncology. Semin Oncol 2019;46:202-9. https://doi.org/10.1053/j.se minoncol.2019.07.001.

9. Kairemo K, Santos EB, Macapinlac HA, Patel S, Conley AP and Hon, DS, et al. Molecular imaging with $3^{\prime}$-deoxy-3'[(18)F]-Fluorothymidine (18F-FLT) PET/CT for early response to targeted therapies in sarcomas: A pilot study. Diagnostics (Basel) 2020;10:125. https://doi.org/10.3390/diagnostics10030125.

10. Gulyás B, Halldin C. New PET radiopharmaceuticals beyond FDG for brain tumor imaging. Q J Nucl Med Mol Imaging 2012;56:17390.

11. Katzenellenbogen JA. The quest for improving the management of breast cancer by functional imaging: The discovery and development of $16 \alpha-[18 \mathrm{~F}]$ fluoroestradiol (FES), a PET radiotracer for the estrogen receptor, a historical review. Nucl Med Biol 2020. h ttps://doi.org/10.1016/j.nucmedbio.2020.02.007.

12. Siebermair J, Koehler M, Kupusovic J, Nekolla S, Guberina N, et al. Cardiac fibroblast activation detected by Ga-68 FAPI PET imaging as a potential novel biomarker of cardiac injury/remodeling. J Nucl Cardiol (current issue).

13. Barrett R, Puré E. Cancer-associated fibroblasts: Key determinants of tumor immunity and immunotherapy. Curr Opin Immunol 2020;64:80-7. https://doi.org/10.1016/j.coi.2020.03.004.

14. Kuzet SE, Gaggioli C. Fibroblast activation in cancer: When seed fertilizes soil (review). Cell Tissue Res 2016;365:607-19. https://d oi.org/10.1007/s00441-016-2467-x.

15. Busek P, Mateu R, Zubal M, Kotackova L, Sedo A. Targeting fibroblast activation protein in cancer: Prospects and caveats. Front Biosci 2018;23:1933-68. https://doi.org/10.2741/4682.

16. Watson GA, Doi J, Hansen AR, Spreafico A. Novel strategies in immune checkpoint inhibitor drug development: How far are we from the paradigm shift? Br J Clin Pharmacol 2020. https://doi. org/10.1111/bcp.14355.

Publisher's Note Springer Nature remains neutral with regard to jurisdictional claims in published maps and institutional affiliations. 